\title{
A CHARACTERIZATION OF OPEN MAPPING IN TERMS OF CONVERGENT SEQUENCES
}

\author{
IRWIN E. SCHOCHETMAN
}

Received 5 March 2005; Revised 4 January 2006; Accepted 22 January 2006

It is certainly well known that a mapping between metric spaces is continuous if and only if it preserves convergent sequences. Does there exist a comparable characterization for the mapping to be open? Of course, the inverse mapping is set-valued, in general. In this research/expository note, we show that a mapping is open if and only if the set-valued inverse mapping preserves convergent sequences in an appropriate set-theoretic sense.

Copyright @ 2006 Hindawi Publishing Corporation. All rights reserved.

\section{Introduction}

Let $X$ and $Y$ be topological spaces and $f: X \rightarrow Y$ a general mapping. In particular, $f$ need not be onto. For convenience, we will assume that $X$ and $Y$ are metric spaces, so that sequences suffice when considering convergence. (We leave the extension of our main result to nets for the interested reader to pursue.) By definition, $f$ is continuous if $f^{-1}(U)$ is open in $X$, whenever $U$ is open in $Y$. It is certainly well known that $f$ is continuous if and only if $\lim _{n \rightarrow \infty} f\left(x_{n}\right)=f(x)$ in $Y$, whenever $\lim _{n \rightarrow \infty} x_{n}=x$ in $X$ (see [1, Theorem 1, page 34]). Also by definition, $f$ is open if $f(U)$ is open in $Y$, whenever $U$ is open in $X$. Does there exist a characterization of openness for $f$ in terms of convergent sequences analogous to that for continuity? It would seem that a positive response to this question should be well known. However, we have not been able to find such a result in the literature. Of course, this question is complicated by the fact that $f^{-1}$ need not be one-to-one in general, in which case we obtain a set-valued mapping from $Y$ into $X$ given by $y \rightarrow f^{-1}(y)$, for all $y \in Y$, where $f^{-1}(y)$ might be empty, for some $y \in Y$. Nevertheless, the answer to the previous question is yes. Specifically, in this research/expository note, we will show that a mapping is open if and only if its set-valued inverse mapping satisfies a familiar subsequence convergence property (see Theorem 2.2). We have found this characterization to be very useful in our research (i.e., see [9]).

Although we have not been able to find our main result in the literature, there do exist comparable results which require additional assumptions. In [3], the authors establish

Hindawi Publishing Corporation

International Journal of Mathematics and Mathematical Sciences

Volume 2006, Article ID 76162, Pages 1-5

DOI 10.1155/IJMMS/2006/76162 
such a result (see Theorem 2.4) for which no proof is given. Moreover, their result is stated in terms of ultranets. However, of greatest importance is the fact that they assume the mapping is continuous. The same is true in [4]. It is shown in [4, Proposition 1, Part 4] that our subsequence convergence condition is equivalent to openness, but under the assumption that the mapping in question is once again continuous and onto. (Moreover, the work [4] is not easy to obtain.) Neither one of the two previous references states the relevant results in the context of a set-valued inverse mapping which is, as we have tried to motivate, a more appropriate way to view openness.

It has been suggested that by combining (in order) [6, Proposition 7.2.9], [1, Lemma 6.2.2] and the fact that limit points are cluster points, one may conclude that the subsequence convergence condition is equivalent to openness of the mapping. However, this argument shows only that the subsequence convergence condition is necessary for openness. Thus, this argument does not establish our main result either. Moreover, we feel that the sufficiency is the more important half of the main theorem since it is more likely to be used to show that a given mapping is open (see [9], e.g.).

Finally, our main result is verified in a practical and familiar context. Our proof is direct and easily accessible to students in first-semester real analysis and point-set topology. The most advanced concept we use is that of set-valued mapping, which easily can be circumvented (as in $[3,4]$ ).

As motivation for what follows, let $\left\{x_{n}\right\}_{n=1}^{\infty}$ be a sequence in $X$. Let $\limsup _{n} f\left(x_{n}\right)$ denote the set of cluster points of the sequence $\left\{f\left(x_{n}\right)\right\}_{n=1}^{\infty}$ in $Y$, that is, the set of points $y$ for which there exists a subsequence $\left\{x_{n_{k}}\right\}_{k=1}^{\infty}$ of $\left\{x_{n}\right\}_{n=1}^{\infty}$ such that the corresponding subsequence $\left\{f\left(x_{n_{k}}\right)\right\}_{k=1}^{\infty}$ of $\left\{f\left(x_{n}\right)\right\}_{n=1}^{\infty}$ converges to $y$. This is common notation for denoting the set of cluster points (see [8, page 337]). Moreover, it is worth noting that $\liminf _{n} f\left(x_{n}\right)$ is used to denote the set of limit points [8, page 335].

We then have the following alternate ways of looking at continuity.

Lemma 1.1. Let $X$ and $Y$ be metric spaces and $f: X \rightarrow Y$ a map. Then the following are equivalent.

(i) $f$ is continuous.

(ii) $f(x) \in \limsup _{n} f\left(x_{n}\right)$, for every convergent sequence $\lim _{n} x_{n}=x$ in $X$.

(iii) $\{f(x)\}=\limsup _{n} f\left(x_{n}\right)$, for every convergent sequence $\lim _{n} x_{n}=x$ in $X$.

Proof. (ii) $\Rightarrow($ i). Suppose $f$ is not continuous. Then there exists a convergent sequence $\lim _{n \rightarrow \infty} x_{n}=x$ in $X$ for which $\lim _{n \rightarrow \infty} y_{n} \neq y$, where $f\left(x_{n}\right)=y_{n}$, for all $n$, and $f(x)=$ $y$. Thus, there exists a convergent subsequence $\lim _{k \rightarrow \infty} x_{n_{k}}=x$ of $\left\{x_{n}\right\}_{n=1}^{\infty}$ and an open neighborhood $U$ of $y$ in $Y$ such that $y_{n_{k}} \notin U$, for all $k$. But, by hypothesis, $f(x) \in$ $\limsup _{k} f\left(x_{n_{k}}\right)$, that is, $y \in \limsup _{k} y_{n_{k}}$. Hence, there exists a convergent subsequence $\lim _{j \rightarrow \infty} y_{n_{k_{j}}}=y$ of $\left\{y_{n_{k}}\right\}_{k=1}^{\infty}$. Hence, $y_{n_{k_{j}}} \in U$ eventually, which is a contradiction, since $y_{n_{k}} \notin U$, for all $k$.

(iii) $\Rightarrow$ (ii). The proof is obvious.

(i) $\Rightarrow$ (iii). Let $\lim _{n \rightarrow \infty} x_{n}=x$ be a convergent sequence in $X$. Since $f$ is continuous, we have that $\{f(x)\} \subseteq \limsup _{n} f\left(x_{n}\right)$. Let $y \in \limsup _{n} f\left(x_{n}\right)$. Then there exists a convergent subsequence $\lim _{k \rightarrow \infty} x_{k}=x$ of $\left\{x_{n}\right\}_{n=1}^{\infty}$ for which $\lim _{k \rightarrow \infty} f\left(x_{n_{k}}\right)=y$. Since $f$ is continuous, $\lim _{k \rightarrow \infty} f\left(x_{n_{k}}\right)=f(x)$. Therefore, $f(x)=y$, since $Y$ is a Hausdorff space, that 
is, the sequence $\left\{f\left(x_{n_{k}}\right)\right\}_{k=1}^{\infty}$ cannot converge to two distinct points in $Y$. Consequently, $\{f(x)\} \supseteq \limsup _{n} f\left(x_{n}\right)$.

\section{The main result}

For our purposes here, we require the notion of the set of cluster points of a sequence of arbitrary sets. Let $\left\{A_{n}\right\}_{n=1}^{\infty}$ be a sequence of nonempty subsets of $X$. Then $x \in X$ is a cluster point of this sequence of sets if there exists a subsequence $\left\{A_{n_{k}}\right\}_{k=1}^{\infty}$ of $\left\{A_{n}\right\}_{n=1}^{\infty}$ and a corresponding convergent sequence $\lim _{k \rightarrow \infty} x_{k}=x$ in $X$ such that $x_{k} \in A_{n_{k}}$, for all $k$. Let $\limsup _{n} A_{n}$ denote the set of all such points (see [8, page 337]). Similarly, $\liminf _{n} A_{n}$ denotes the set of limit points of the $A_{n}$, that is, those $x \in X$ such that $x=\lim _{n} x_{n}$, for $x_{n} \in A_{n}$, for all $n$ (see [8, page 335]). We then have the following property (amongst others). We emphasize that the sets $A_{n}$ are not assumed to be closed.

Lemma 2.1. In general, $\lim \sup _{n} A_{n}$ is a closed subset of $X$.

Proof. It can be shown that $\lim \sup _{n} A_{n}=\cap_{n=1}^{\infty} \overline{\cup_{k \geq n} A_{k}}$ (see [8, page 337]). See also [2, page 121] and [5, page 169].

The following is our main result.

Theorem 2.2. Let $X$ and $Y$ be metric spaces and let $f: X \rightarrow Y$ be a map. Then the following are equivalent.

(i) $f$ is open.

(ii) $f^{-1}(y) \subseteq \limsup _{n} f^{-1}\left(y_{n}\right)$, for every convergent sequence $\lim _{n} y_{n}=y$ in $Y$.

Proof. (i) $\Rightarrow$ (ii). Suppose $f$ is open and $\left\{y_{n}\right\}_{n=1}^{\infty}$ is a sequence in $Y$ which converges to $y \in Y$. Since $f$ is open, the range $f(X)$ of $f$ is an open subset of $Y$. Either $y \in f(X)$ or not. If $y \notin f(X)$, then $f^{-1}(y)=\varnothing$, so that part (ii) holds. If $y \in f(X)$, then $y_{n}$ is eventually in $f(X)$, since it is open. Thus, without loss of generality, we may assume $y_{n} \in f(X)$, for all $n$. The subset $\limsup _{n} f^{-1}\left(y_{n}\right)$ of $X$ is clearly closed (see Lemma 2.1). Suppose the subset $f^{-1}(y)$ of $X$ is not contained in $\limsup _{n} f^{-1}\left(y_{n}\right)$. Then there exists $x \in$ $f^{-1}(y)$, that is, $f(x)=y$, such that $x \notin \limsup _{n} f^{-1}\left(y_{n}\right)$. Let $U$ denote the complement of $\limsup _{n} f^{-1}\left(y_{n}\right)$ in $X$, an open neighborhood of $x$. Let $\left\{U_{n}\right\}_{n=1}^{\infty}$ be a descending open base for the neighborhood system of $\{x\}$, which is contained in $U$, that is, a sequence of neighborhoods $U_{n}$ of $x$ in $X$ satisfying

$$
\begin{gathered}
x \in U_{n+1} \subseteq U_{n} \subseteq U \quad \forall n, \\
\cap_{n=1}^{\infty} U_{n}=\{x\} .
\end{gathered}
$$

Let $n=1$. Then, by hypothesis, $f\left(U_{1}\right)$ is an open neighborhood of $f(x)=y$ in $Y$. Since $\lim _{n} y_{n}=y$, there exists $n_{1}$ such that $y_{n} \in f\left(U_{1}\right)$, for all $n \geq n_{1}$. Suppose we have chosen $y_{n_{1}}, \ldots, y_{n_{k}}$ such that $y_{n_{j}} \in U_{j}$, for all $j=1, \ldots, k$, and $y_{n} \in f\left(U_{k}\right)$, for all $n \geq n_{k}$. For $U_{k+1}$, there exists $n_{k+1}>n_{k}$ such that $y_{n} \in f\left(U_{k+1}\right)$, for all $n \geq n_{k+1}$. Then $\left\{y_{n_{k}}\right\}_{k=1}^{\infty}$ is a subsequence of $\left\{y_{n}\right\}_{n=1}^{\infty}$. Let $x_{k} \in X$ be such that $x_{k} \in U_{k}$, and $f\left(x_{k}\right)=y_{n_{k}}$, that is, $x_{k} \in f^{-1}\left(y_{n_{k}}\right)$, for all $k$. Then the sequence $\left\{x_{j}\right\}_{j=1}^{\infty}$ is eventually in each $U_{k}$. In fact, given 
$k$, we have $x_{k} \in U_{k}$, and $x_{j} \in U_{j} \subseteq U_{k}$, for all $j \geq k$. Consequently, $\lim _{k \rightarrow \infty} x_{k}=x$. By definition of $\limsup _{n} f^{-1}\left(y_{n}\right)$, it follows that $x \in \limsup _{n} f^{-1}\left(y_{n}\right)$, a contradiction. Thus,

$$
f^{-1}(y) \subseteq \limsup _{n} f^{-1}\left(y_{n}\right)
$$

(ii) $\Rightarrow$ (i). Suppose the condition in (ii) holds and $f$ is not open. Then there exists an open subset $U$ of $X$ such that $f(U)$ is not open in $Y$, that is, its complement $Y \backslash f(U)$ is not closed in $Y$. Consequently, there exists a convergent sequence $\lim _{n \rightarrow \infty} y_{n}=y$ in $Y$ such that $y_{n} \notin f(U)$, for all $n$, while $y \in f(U)$. Since $y \in f(U)$, there exists $x \in U$ such that $f(x)=y$, that is, $x \in f^{-1}(y)$. By hypothesis,

$$
f^{-1}(y) \subseteq \limsup _{n} f^{-1}\left(y_{n}\right) .
$$

Thus, there exists a subsequence $\left\{y_{n_{k}}\right\}_{k=1}^{\infty}$ of $\left\{y_{n}\right\}_{n=1}^{\infty}$, and a corresponding sequence $\left\{x_{k}\right\}_{k=1}^{\infty}$ in $X$ such that $x_{k} \in f^{-1}\left(y_{n_{k}}\right)$, for all $k$, and $\lim _{k \rightarrow \infty} x_{k}=x$. Then $x_{k} \notin U$, for all $k$; if not, $x_{k} \in U$, for some $k$, which implies

$$
y_{n_{k}}=f\left(x_{k}\right) \in f(U),
$$

a contradiction. Since $X \backslash U$ is closed, and $x_{k} \in X \backslash U$, for all $k$, it follows that $x \in X \backslash U$, that is, $x \notin U$, a contradiction.

As expected, we have the following special case.

Corollary 2.3. If $f$ is one-to-one and onto, then $f$ is open if and only if $f^{-1}$ is continuous. Proof. This follows from Theorem 2.2 and Lemma 1.1.

Remark 2.4. (1) We urge the reader to compare part (ii) of Lemma 1.1 for continuous maps with part (ii) of Theorem 2.2 for open maps. (2) In [2], the author gives three notions of semicontinuity and continuity for set-valued maps, each of which is a generalization of classical continuity for single-valued maps. One of these is part (ii) of our main theorem for set-valued inverse mappings. Thus, our theorem says that a pointvalued mapping $f$ is open if and only if the corresponding set-valued inverse mapping $f^{-1}$ is continuous (in the sense of part (ii) of the main theorem). (3) The results contained herein can be reworked for continuity and openness at a point. The details are straightforward and left to the reader.

\section{Acknowledgment}

The author wishes to thank the two referees for some helpful comments and suggestions which have significantly improved the exposition.

\section{References}

[1] G. Beer, Topologies on Closed and Closed Convex Sets, Mathematics and Its Applications, vol. 268, Kluwer Academic, Dordrecht, 1993.

[2] C. Berge, Topological Spaces, Oliver and Boyd, London, 1963. 
[3] M. M. Clementino and D. Hofmann, Triquotient maps via ultrafilter convergence, Proceedings of the American Mathematical Society 130 (2002), no. 11, 3423-3431.

[4] O. Hájek, Notes on quotient maps, Commentationes Mathematicae Universitatis Carolinae 7 (1966), 319-323.

[5] F. Hausdorff, Set Theory, Chelsea, New York, 1957.

[6] E. Klein and A. C. Thompson, Theory of Correspondences, Canadian Mathematical Society Series of Monographs and Advanced Texts, John Wiley \& Sons, New York, 1984.

[7] A. N. Kolmogorov and S. V. Fomin, Elements of the Theory of Functions and Functional Analysis. Vol. 1. Metric and Normed Spaces, Graylock Press, New York, 1957.

[8] K. Kuratowski, Topology. Vol. I, Academic Press, New York, 1966.

[9] I. E. Schochetman and R. L. Smith, Existence of efficient solutions in infinite horizon optimization under continuous and discrete controls, Operations Research Letters 33 (2005), no. 1, 97-104.

Irwin E. Schochetman: Department of Mathematics and Statistics, Oakland University, Rochester, MI 48309, USA

E-mail address: schochet@oakland.edu 


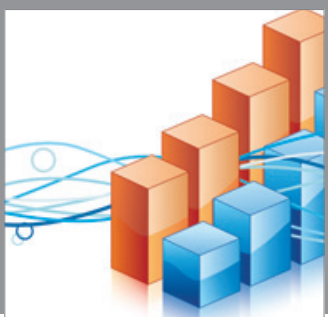

Advances in

Operations Research

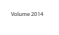

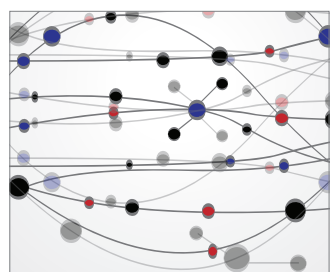

\section{The Scientific} World Journal
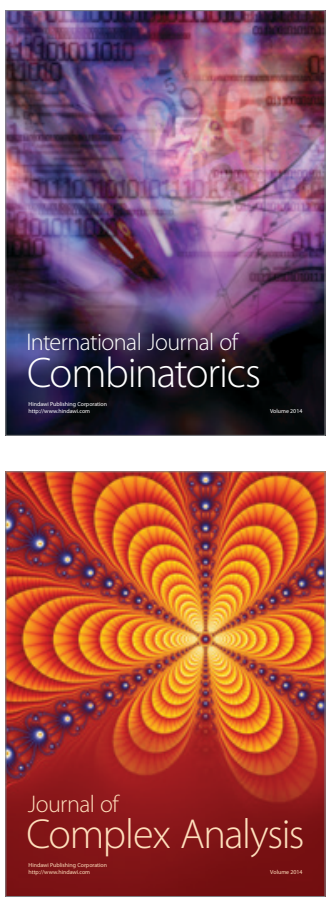

International Journal of

Mathematics and

Mathematical

Sciences
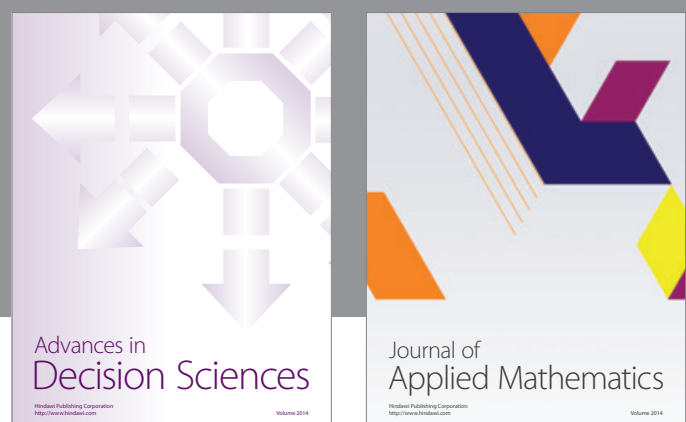

Journal of

Applied Mathematics
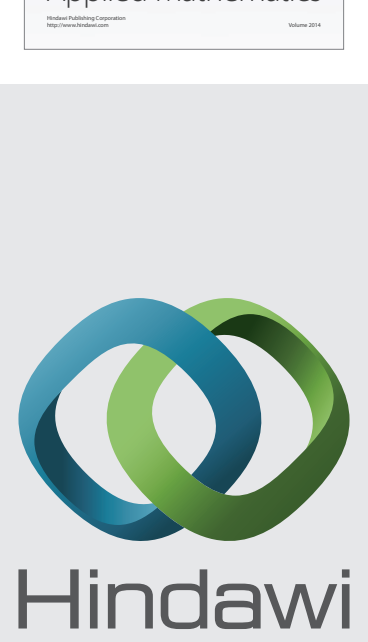

Submit your manuscripts at http://www.hindawi.com
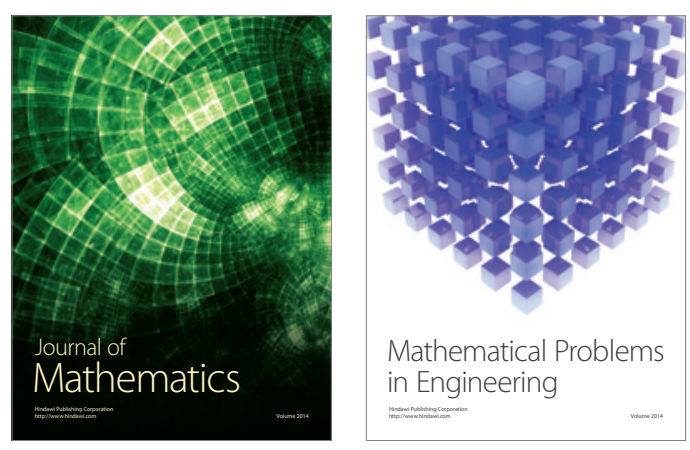

Mathematical Problems in Engineering
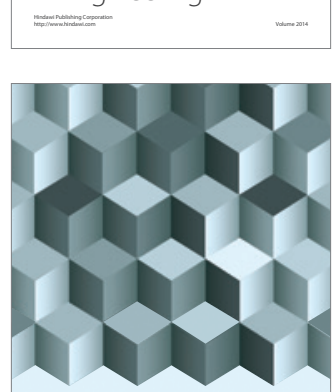

Journal of

Function Spaces
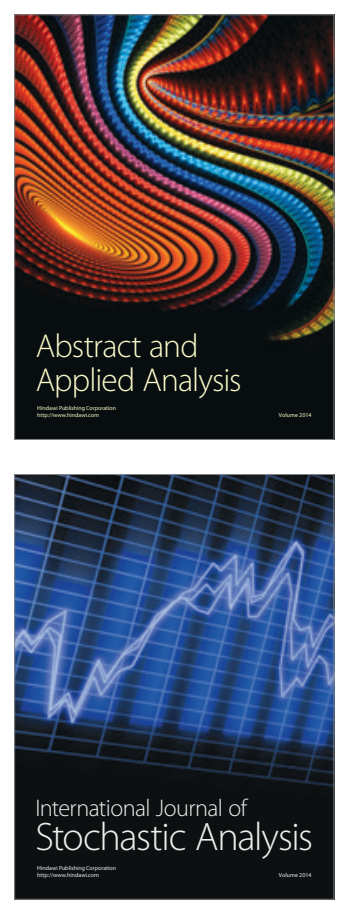

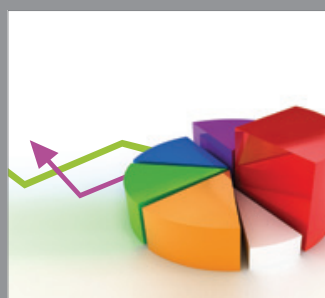

ournal of

Probability and Statistics

Promensencen
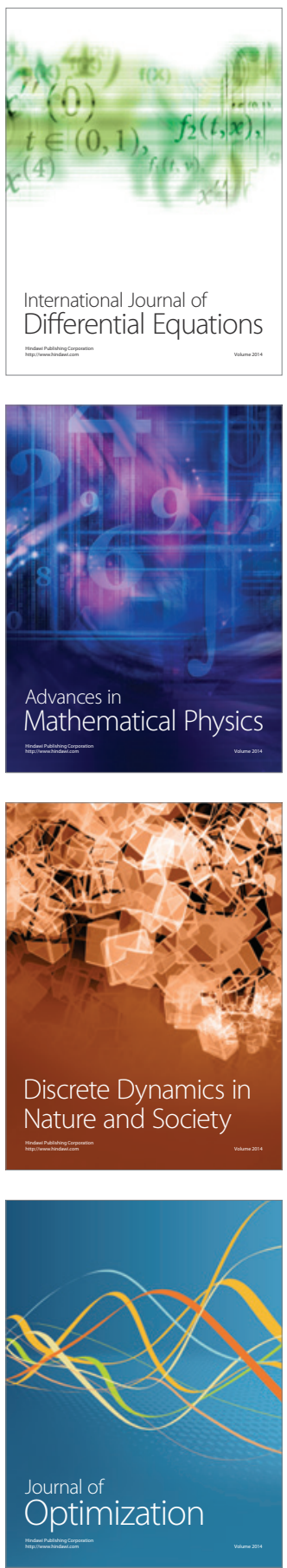